\title{
5G, Blockchain and IPFS: A General Survey with Possible Innovative Applications in Industry 4.0
}

\author{
Ivan Jovović ${ }^{1}$, Siniša Husnjak ${ }^{1}$, Ivan Forenbacher ${ }^{1}$, Sven Maček ${ }^{1}$ \\ \{ ijovovic@fpz.hr, shusnjak@fpz.hr, iforenbacher@fpz.hr, sven.macek@gmail.com \} \\ University of Zagreb, Faculty of Transport and Traffic Sciences, \\ Department of Information and Communication Traffic, \\ Zagreb, Republic of Croatia, EU
}

\begin{abstract}
The Industry 4.0 is experiencing significant challenges, including the need for an increased amount of data transmission with improved security, transparency and credibility. The 5th Generation Mobile Network (5G) and Blockchain are innovative emerging technologies that can respond to these needs. 5G will enable extremely large channel capacities and reduce data latency, while Blockchain's innovative data-sharing mode of operation delivers an improved high level of security, transparency, and credibility of stored data. Therefore, this paper presents a general survey of the potential application of 5G network and Blockchain technology in Industry 4.0. The results may be used by first-movers firms for gaining technological leadership on the market.
\end{abstract}

Keywords: Industry 4.0, Internet of Things (IoT), 5G mobile network, Blockchain, IPFS

\section{Introduction}

Innovative and future emerging technologies (FET) are generator of dynamic changes in virtually all areas. One of the areas undergoing an intensive transition is industrial production. The term Industry 4.0 describes significant transformation of current industrial concept leading to the novel one, primarily based on the progressive growth of information and communication technologies (ICT). According to [1], Industry 4.0 can be described as a platform for integrating ICT technology and manufacturing systems.

The need of Industry 4.0 is to turn regular devices into self-aware and self-learning devices to improve overall management processes while maintaining interaction with the environment. Real-time data monitoring, product status tracking, product location and product management process are the main industry needs of Industry 4.0 [2].

The concept of Industry 4.0 takes into account increased use of information technology in manufacturing processes where physical objects are simply integrated with the information and communication network. Therefore, concepts of Internet of Things (IoT) and Internet of Services (IoS) are already being considered cornerstones of Industry 4.0. Importantly, the fifth generation mobile network $(5 \mathrm{G})$ will enable the implementation of the IoT concept. $5 \mathrm{G}$ is the term used to describe the next (fifth) generation of wireless networks and represents the evolution of existing access radio technologies.

$5 \mathrm{G}$ will not only be the evolution of the broadband network, but a unique network with completely new features, as well. These features will provide the user with a number of performance enhancements in terms of network capacity increase, shorter latency, more 
mobility and increased network reliability and security. This, in turn, will result in allconnected environment, called IoT [3].

There are many major challenges in the implementation of IoT concept, such as security, privacy, trust, and limited network capacity. This is due to the need of transferring a very large amount of data in order to provide the user with an adequate service or access to information. [4].

Some of the challenges and fundamental issues arising from the implementation of Industry 4.0, according to [5], are given as:

- today's communications network cannot provide enough large bandwidth for demanding communications and the need for large amount of data transfer

- dramatically increased need to protect critical industrial systems, their data and production lines from cyber threats

Therefore, the goal of this paper is to provide general insight of contemporary technologies like $5 \mathrm{G}$ and Blockchain which could lead to innovative applications in Industry 4.0.

In contrast to previous generations of mobile networks, $5 \mathrm{G}$ network has one of the most important features - the ability to transfer large amounts of data with reduced latency. This is particularly important for devices which continuously require information in real-time. In this context, along with the additional features described below, 5G network ensures the needs of Industry 4.0 to connect an enormous number of IoT devices, and for increased volume of generated data traffic.

On the other hand, Blockchain technology with its innovative working principle in the form of distributed data storage systems provides an enormously high level of security, transparency, credibility and immutability of stored data.

\section{Industry 4.0 and IoT}

Industry 4.0 is the umbrella concept for a new industrial paradigm that includes a number of future industry achievements related to Cyber-Physical Systems (CPS), IoT, IoS, Robotics, Big Data, Cloud Manufacturing and Augmented Reality. Adopting these technologies is key to the development of more intelligent manufacturing processes, including devices, machines, production modules, and products that are able to independently exchange information, stimulate actions and control each other, thus enabling an intelligent manufacturing environment [2], [6].

One of the more important terms coming along with Industry 4.0 is IoT. The position of industrial production is related to the integration of ICT (Information and Communication Technology) systems with production processes, internal and external facilities, network of suppliers and customers, machine-to-machine communication (M2M) and communication with employees [7]. Integration of IoT and IoS into the production environment introduces 4th industrial evolution.

An important feature of Industry 4.0 is the ability to collect and use large amounts of data [1]. Connected vehicles, smart manufacturing, global logistics tracking, smart agriculture, smart metrics and other applications are some of the first but also promising areas of the IoT domain that deserve special attention. The above mentioned applications will be developed rapidly with the advent of $5 \mathrm{G}$ network [8]. 


\section{Industry 4.0 and $5 G$}

In the manufacturing industry, the digitalization of processes and workflows is creating requirements such as low latency, real-time performance, mobility, enhanced security, and ultra-reliable and very high availability [9]. 5G networks will provide significant increase of channel capacity compared to the previous generations of mobile networks. Furthermore, 5G networks will reduce latency and improve network efficiency. A well-designed network architecture will provide latency from end to end to less than $5 \mathrm{~ms}$. This will enable ultrareliable low latency communication for M2M communications [8].

The table 1 below shows the technical capabilities under IMT-2020 radio interface(s) as improvements vis-à-vis its predecessor in terms of peak data rates, spectral efficiency, mobility, latency, energy efficiency and connection density, among others.

Table 1: Key minimum requirements related to technical performance for IMT-2020 radio interface(s), [10]

\begin{tabular}{ll}
\hline Technical capability & Minimum requirement \\
\hline Peak data rate & $1-20 \mathrm{Gbit} / \mathrm{s}$ \\
User experienced data rate & $10-100 \mathrm{Mbit} / \mathrm{s}$ \\
Peak spectral efficiency & $15-30 \mathrm{bit} / \mathrm{s} / \mathrm{Hz}$ \\
Mobility & $350-500 \mathrm{~km} / \mathrm{h}$ \\
Latency & $1-10 \mathrm{~ms}$ \\
Connection density & $10000-1000000$ devices $/ \mathrm{km} 2$ \\
Network energy efficiency & $90 \%$ more efficient \\
Area traffic capacity & $0.1-10 \mathrm{Mbit} / \mathrm{s} / \mathrm{m} 2$ \\
\hline
\end{tabular}

Although the final requirements for $5 \mathrm{G}$ have not been completed yet, there is a common understanding of the main use-case scenarios and applications that $5 \mathrm{G}$ could support. Three major use-cases, according to [10] and [11] are:

- massive mobile connectivity that would enable enhanced mobile broadband (eMBB);

- connectivity of millions of devices, that would enable massive machine type communication (mMTC); and

- resilient, instantaneous connectivity, that would enable ultra-reliable and low latency communications (URLLC).

Most use cases enabled by cellular networks will reduce operational costs in a factory [9]. Full implementation of Industry 4.0 will be achieved by launching 5G mobile network since real-time communication between machines is of crucial importance, requiring a reduction in latency to be less than $1 \mathrm{~ms}$, allowing smooth operation of machines in all branches of industry. Table 2 shows application of $5 \mathrm{G}$ in Industry 4.0 according to a particular field of application.

Table 2: Application of 5G in Industry 4.0 across different fields [8]

\begin{tabular}{lll}
\hline eMBB & mMTC & uRLLC \\
\hline Wireless Industry Camera & Status Monitoring & Wireless Cloud PLC \\
Industrial Sensors & Asset Tracking & Synchronized Robotics \\
Remote Control & Cloud Based Automated Guided Vehicles & \\
Edge Computing Analytics & Logistics \& Inventory Monitoring & \\
\hline
\end{tabular}


$5 \mathrm{G}$ networks must also provide a flexible platform for new services such as connecting a large number of IoT devices and critical communications between them [12]. Connectivity requirements in a factory are very dependent on the use case and application, and so the network also needs to support service differentiation. Some use cases are possible to address with evolved 4G (LTE) technology, while others such as production and robot control may require latency between 1 and 10 milliseconds, which can only be achieved with 5G technology [9].

\section{Decentralized data storage system}

In everyday processes involved, the emphasis is on „trust” among all involved parties. This fact is taken for granted and virtually not noticed in electronic communications. For example, when buying a product there is a trust between the buyer and the seller. The buyer believes that he actually gets the original product, with declared quality and features, and the seller that buyer will actually pay for the purchased product and will not use counterfeit money.

In general, systems can be divided into centralized and decentralized. Centralized systems are those in which there is a „trusted“ central server deciding on everything - from storing data, to guaranteeing data integrity, and validating client requirements for data.

On the other hand, the main characteristic of decentralized systems is peer-to-peer communication that results in elimination of mediators while simultaneously increasing the level of security, trust and data integrity. More importantly, in such system there is no central point of failure.

\subsection{Blockchain - decentralized system of equal partners}

Blockchain is an innovative technology that will, according to Gartner, reach its full potential for 5-10 years [13]. It consists of global architectural and management decentralization because there is no infrastructure central point of failure and management, as well as logical centralization because the system behaves like a „big computer“.

It is also called distributed shared ledger because the transaction database is located on all nodes in the network [14], [15]. Transactions are used to exchange digital assets - money, personal or financial data, health status or records of a machine's operation or any other digital asset that can be transmitted. Transaction records are stored in the block. Blocks are then linked to the chain by using hash values, thus making a blockchain and providing maximum integrity of data. Each new block is linked to the previous one (Figure 1).

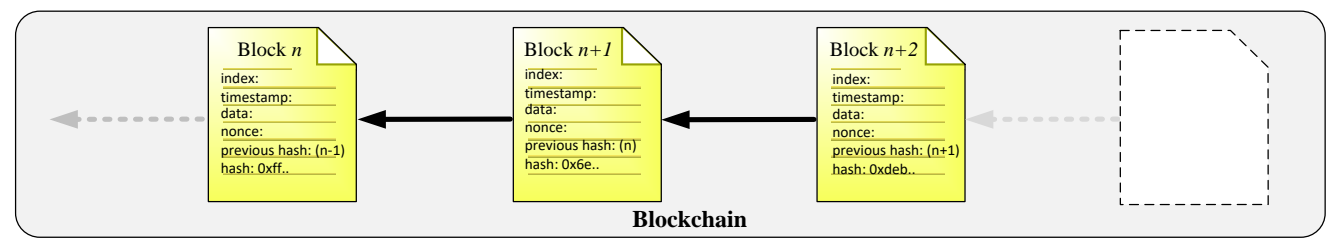

Fig. 1. Blockchain concept

One of the most important characteristics of blockchain is a decentralized consensus that serves for reaching agreement about the validity of each transaction between all nodes in the network before it is stored in block (Figure 1). This is possible because blockchain systems 
use the principle of equal partners. Each of the participating partners in the system is responsible for broadcasting and validating new transactions and writing them into new blocks. This enables secure, transparent, immutable and credible data exchange where nodes take on the role of the current central point in centralized networks (Figure 2). At the same time, each node has its own current copy of blockchain. Therefore, the data stored in the blockchain cannot be altered because a consensus is needed from all other nodes in the network.

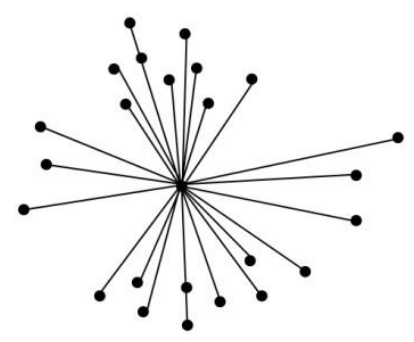

a)

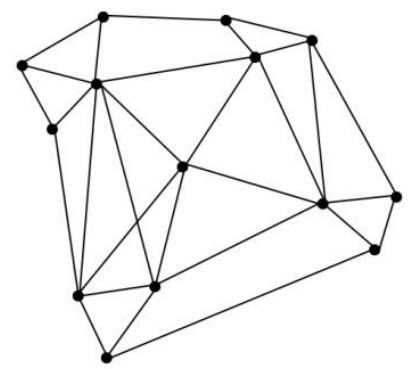

b)

Fig. 2. Architectural comparison of traditional (a) and blockchain (b) system

Blockchain modus operandi can be summarized in the following steps:

1 User $\mathrm{A}$ initiates a request for digital asset transaction to user B, which includes the address of the recipient, the sender, and the subject of the transaction.

2 The transaction request is broadcasted by all peers in the network of the specific blockchain.

3 Validation of the request is performed by peers in the network which are also called miners.

4 Transaction is stored in the block, hash value is calculated for that block, thus ensuring the immutability of the data.

5 User B receives digital assets that were sent from user A.

\subsection{Smart contracts}

In order to explain the importance of smart contracts, it is necessary to draw an analogy with today's traditional ways of executing contracts that most often involve multiple mediators, including lawyers, notaries, financial institutions and public services. This can result in a complex and time-consuming process. To speed up and simplify the whole process, it is possible to eliminate these intermediaries by applying a smart contract.

Smart contracts, according to [16], are „data driven code that can represent verifiable application logic and help automate a system's rule set". This code is written to the blockchain and executed at any node in the network when specific conditions are met. These terms may be the date, location, user or machine identity, etc. With a smart contract, interested parties can exchange assets easily by using a blockchain network node that contains an application for its execution [17]. 
Figure 3 shows the process of creating smart contracts by following these steps:

1. Possible digital asset exchange between two or more users is written as a program code and stored on a blockchain.

2. Fulfilling the terms defined by a smart contract serves as a trigger for executing contracts by peers and performing a transaction according to the rules defined in the code.

3. Executing a smart contract results in certain changes in the status of a sender or recipient. For example, at the moment of delivery of the product to the address, a smart contract transfers funds from user A to user B that need to be stored in the blockchain.

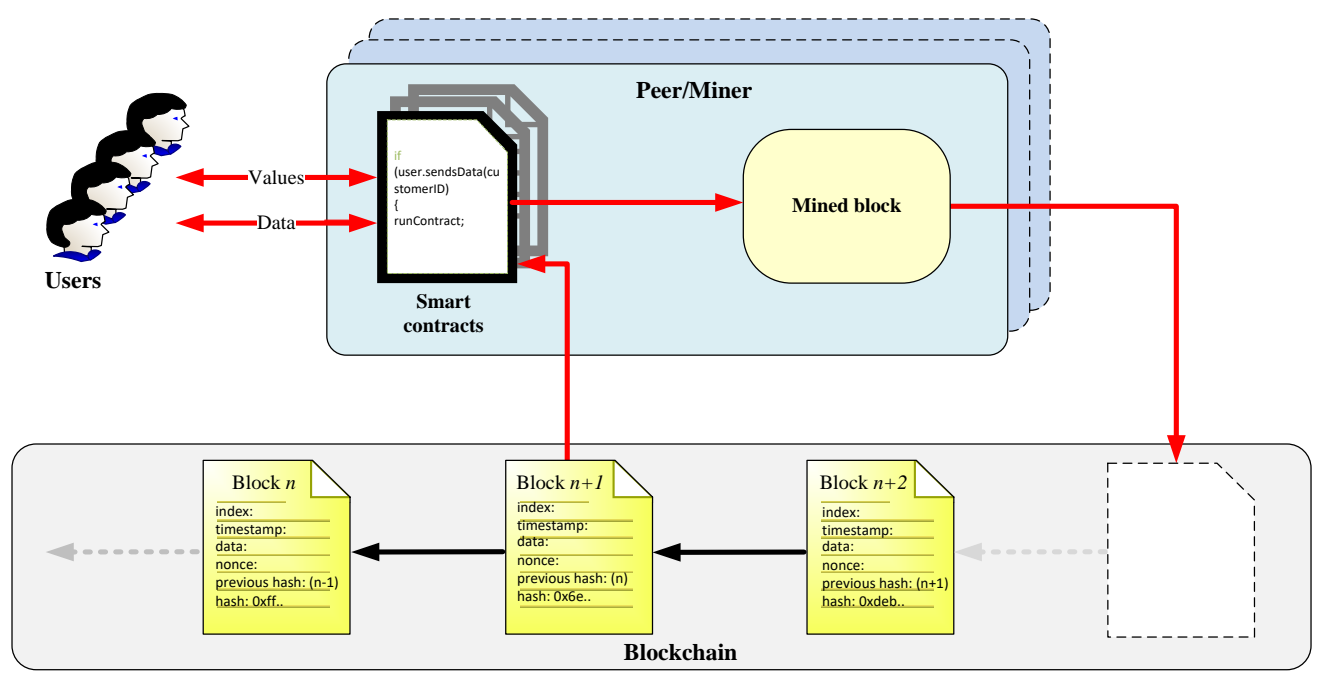

Fig. 3. How a smart contract works [17]

There are various benefits of using smart contracts but reducing transaction costs and increased transparency are the two key elements achieved by increasing digital efficiency with eliminating the need for a middle-man.

\subsection{InterPlanetary File System}

In a blockchain, it is not desirable to store a large amount of data such as documents and multimedia content, as this would significantly increase the processing time.

However, the InterPlanetary File System (IPFS) can be used - an innovative peer to peer distributed data system of equal partners that aims to connect all computers to a single data system. IPFS is a combination of multiple technologies inspired from BitTorrent and Gita, such as Distributed Hash Tables (DHT), Block Exchange System and Version Control System [18].

DHT serves to co-ordinate and maintain metadata on peer-to-peer system. In other words, it is necessary to map the hash values to the objects they represent. When storing an object 
like a file, an IPFS generates a hash value that starts with a prefix Qm and serves for referencing to a specific object. Objects larger than $256 \mathrm{kB}$ are divided into smaller blocks up to $256 \mathrm{kB}$. Then a hash tree is used to interconnect all the blocks that are a part of the same object. IPFS uses Kamdelia DHT [18].

The Block Exchange System is used to exchange blocks using BitTorrent inspired protocols, called BitSwap. It is based on the „market” principle where peers exchange blocks. Peers search for blocks from other peers (,want” list) and offer theirs in exchange („offer” list) [18].

To prevent unauthorized use of stored content on IPFS, it is possible to use asymmetric encryption. The operating principle of IPFS is shown in Figure 4.

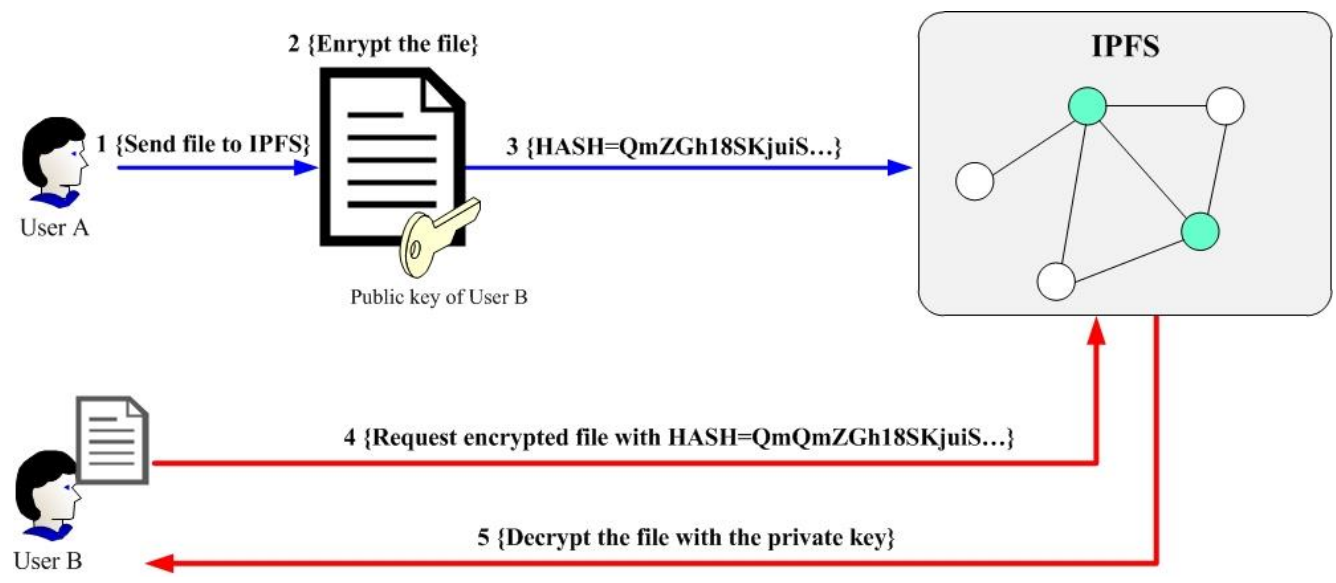

Fig. 4. Storing content in IPFS

It is possible to integrate IPFS and blockchain by storing the desired content, such as a machine's operating data file, into IPFS, and then the generated hash address is stored in the blockchain (Figure 5). This results in increased transparency, i.e. the ability to retrospectively review the content and confidence in its credibility.

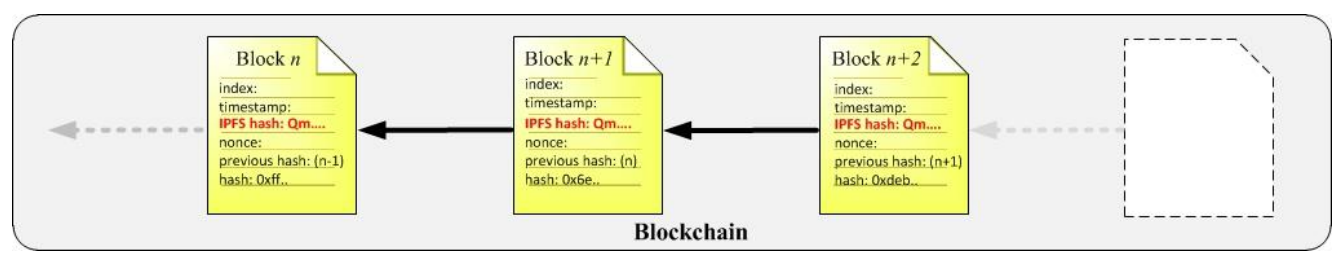

Fig. 5. Integration of IPFS and Blockchain 


\section{Integration of Industry 4.0 and decentralized data storage systems}

Although blockchain is quite a new technology, it can be widely used in industry with many use cases in the new solutions that are constantly introduced. A survey conducted by the World Economic Forum in 2015 predicts that 10\% of global GDP will be stored in blockchain technology by 2027. Table 3 shows cases of blockchain technology use in various branches of industry [19].

Table 3: Review of blockchain use cases in various industries [19]

\begin{tabular}{ll}
\hline Use cases & Description \\
\hline Crypto currency & - Use of digital money for payment purposes \\
Smart contracts & - Implementation of confidential smart contracts \\
Crowdfunding & - Direct transfer of capital to investments \\
Energy markets & - Local trading of solar energy; Sharing energy among communal \\
& services; Automatic payment for using charging stations for electric \\
& cars \\
Smart assets & - Transfer of ownership rights, such as land ownership; Storage of \\
& intangible assets, such as intellectual property, wills, artwork and other \\
\hline
\end{tabular}

Growing adoption of innovative future and emerging technologies and the increasing focus of key players on the research and development activities are expected to fuel the growth of the Industry 4.0. The international market for Industry 4.0 is anticipated to be bolstered by the demand for a range of technologies, including decentralised data management systems such as blockchain with the case study examples (Table 4).

Table 4: Review of blockchain use cases in Industry 4.0

\begin{tabular}{ll}
\hline Use cases & Description \\
\hline Product status and condition & $\begin{array}{l}\text { - Blockchain allows transparency with a shared record of ownership and } \\
\text { location of parts and products in real time }\end{array}$ \\
Anti-counterfeit solutions & $\begin{array}{l}\text { - Blockchain verifies counterfeit products, fraudulent transactions, } \\
\text { stolen merchandise and diverted goods }\end{array}$ \\
Food production & $\begin{array}{l}\text { - Blockchain improves transparency and efficiency of finding out what } \\
\text { food and where might be contaminated throughout the supply chain }\end{array}$ \\
Infrastructure management & $\begin{array}{l}\text { - Blockchain guarantees transparency into the operation of the network } \\
\text { by knowing the condition or key performance indicators of any }\end{array}$ \\
Production transparency & $\begin{array}{l}\text { - Blockchain ensures the sourcing of materials, production of products, } \\
\text { provides chain-of-custody and certification of supply chains }\end{array}$ \\
\hline
\end{tabular}

As presented in Table 4 it is possible to differentiate various use case scenarios of using blockchain technology in Industry 4.0. Those use case scenarios emphasize a possibility that blockchain could become a key technology to transform many industries. Blockchain technology is also applicable to upgrade current Industry 4.0 business environment by 
increasing the level of trust among partners in the Industry 4.0 value chain. For example, by sharing the private and protected data between all business partners involved.

\section{Conclusion}

Industry 4.0 faces various challenges. Some of the most significant are related to the increased amount of data transfer and ensuring security. This paper presents the features and possibilities of applying novel technologies, such as 5G, blockchain and IPFS that can be used to mitigate above-mentioned challenges.

Since Industry 4.0 is essentially based on the principles of connecting IoT devices, the paper highlights the potential of implementing $5 \mathrm{G}$ network in the context of the IoT ecosystem. Given the increasing number of IoT devices, their functionality and upgrading service offerings, there is a need to increase data channel capacity and reduce latency. The 5G network with its functionalities ensures the industry's 4.0 needs for connectivity of the IoT devices.

In addition to the communication segment, it's necessary to ensure secure data storage. Blockchain, along with IPFS, can certainly respond to such requirements within Industry 4.0. The main advantage is decentralized data storage while ensuring the credibility and the immutability of stored data, thus eliminating the need for middle-man. Therefore, these technologies can prove to be a significant complement to Industry 4.0, primarily because they are expected to reach full potential for 5-10 years. As a result, first-movers firms may gain competitive advantage if they timely identify innovative Blockchain use-cases within Industry 4.0.

Therefore, this paper provided an overview of potential practical use cases of $5 \mathrm{G}$ network and the concept of Blockchain technology in Industry 4.0 ecosystem. Relevant features of these technologies which can improve capabilities with respect to existing solutions offer answers to the diverse challenges of industry 4.0. Answers primarily relate to the amount of data transmitted and reduced latency, as well as ensuring transparency, security and credibility of processes and services within Industry 4.0.

\section{References}

[1] Meissner, H., Ilsen, R., Aurich, J. C.: Analysis of Control Architectures in the Context of Industry 4.0. Procedia CIRP. Vol. 62, pp. 165-169 (2017)

[2] Vaidya, S., Ambad, P., Bhosle, S.: Industry 4.0 - A Glimpse. Procedia Manufacturing. Vol. 20, pp. 233-238 (2018)

[3] 5G PPP, European Commission: The 5G Infrastructure Public Private Partnership (5G PPP) - the next generation of communication networks and services. pp. 5-8. European Commission, EU (2015)

[4] Jovovic, I., Forenbacher, I., Perisa, M.: Massive Machine-Type Communications: An Overview and Perspectives Towards 5G. The 3rd International Virtual Research Conference in Technical Disciplines - RCITD 2015, Vol. 3, pp. 26-33. Zilina, Slovakia (2015)

[5] Wang, S., Wan, J., Li, D., Zhang, C.: Implementing Smart Factory of Industrie 4.0: An Outlook. International Journal of Distributed Sensor Networks. Vol. 12., pp. 1-10. (2016)

[6] Pereira, A. C., Romero, F.: A review of the meanings and the implications of the Industry 4.0 concept. Procedia Manufacturing. Vol. 13, pp. 1206-1214 (2017) 
[7] Smit, J., Kreutzer, S., Moeller, C., Carlberg, M.: Policy Department A: Economic and Scientific Policy - Industry 4.0. pp. 1-94. European Parliament, EU (2016)

[8] Wang, A., Yu, L., Mudesir, A., Zhu, D., Zhao, B., Siew, T. S.: 5G Unlocks a World of Opportunities: Top Ten 5G Use Cases. Huawei Technologies Co., Shenzen, China (2017)

[9] Jejdling, F.: Ericsson Mobility Report - June 2018. Ericsson, Stockholm, Sweden (2018)

[10] International Telecommunication Union (ITU). Minimum requirements related to technical performance for IMT-2020 radio interface(s). Radiocommunication Sector of ITU - ITU-R. pp. 1-9 Geneva, Switzerland (2017)

[11] DotEcon, Axon Partners. Study on Implications of 5G Deployment on Future Business Models. Vol. 18, pp. 1-116. US (2018)

[12] Suthar, P.: 5G Technology Components - Building blocks of 5G networks. Nokia, Helsinki, Finland (2017)

[13] Paneta, K.: Top Trends in the Gartner Hype Cycle for Emerging Technologies, 2017. Gartner, Stamford, US (2017)

[14] Pazaitis, A., De Filippi, P., Kostakis, V.: Blockchain and value systems in the sharing economy: The illustrative case of Backfeed. Technological Forecasting and Social Change. Vol. 125, pp. 105115 (2017)

[15] Turk, Ž., Klinc, R.: Potentials of Blockchain Technology for Construction Management. Procedia Engineering. Vol. 196, pp. 638-645 (2017)

[16] Huckle, S., Bhattacharya, R., White, M., Beloff, N.: Internet of Things, Blockchain and Shared Economy Applications. Procedia Computer Science. Vol. 58, pp. 461-466 (2016)

[17] Eze, P. U., Eziokwu, T., Okpara C. R.: A Triplicate Smart Contract Model using Blockchain Technology. Circulation in Computer Science - Disruptive Computing, Cyber - Physical Systems (CPS), and Internet of Everything (IoE). pp. 1-10 (2017)

[18] Benet, J. B.: IPFS - Content Addressed, Versioned, P2P File System. CoRR, vol. abs/1407.3, no. Draft 3, 2014

[19] Dieterich, V., Ivanovic, M., Meier, T., Zäpfel, S., Utz, M., Sandner, P.: Application of Blockchain Technology in the Manufacturing Industry. pp. 1-23. Frankfurt School Blockchain Center, Germany (2017) 